\title{
Finger Vein Recognition Using Log Gabor Filter and Local Derivative Pattern
}

\author{
Lin Feng, Wang Chao* and Peng Jialiang \\ Information and Network Administration Center, Heilongjiang University, Harbin \\ 150080, China \\ linfeng@hlju.edu.cn, ${ }^{*}$ wangchao@hlju.edu.cn,peng jialiang@hlju.edu.cn
}

\begin{abstract}
Finger vein recognition is a promising personal identification technology due to its advantages over other existing biometric recognition. In this paper, a novel finger vein recognition method is proposed based on Log Gabor filter and Local Derivative Pattern. Through combining Log Gabor convolution magnitude and Local Derivative Pattern operator, the extracted finger vein feature vector is more discriminative. Blocked Principal Component Analysis algorithm is further applied to reduce the dimension of extracted feature vectors and improve recognition performance. The experimental results show that the proposed approach significantly outperforms other similar approaches.
\end{abstract}

Keywords: Finger vein recognition, Log Gabor filter, Local Derivative Pattern

\section{Introduction}

As a new biometric technology, finger vein recognition begins to be adapted to the increasing demand of personal identification in current society. Compared to other traditional biometric characteristics (such as face, iris, fingerprints, etc.), finger vein has the following advantages [1]. (1) Contact-less: the imaging procedure of finger vein requires near-infra-red (NIR) illumination to extract the vascular structures residing inside fingers by a non-contact way. (2) Live-ness: finger vein pattern can only be imaged and recognized on living fingers with blood flow, thereby offering strong anti-spoofing capabilities. (3) Security: finger vein is the hidden structure that is extremely difficult to be stolen with a high degree of privacy, and the personal identification based on finger vein is immune to fake fingers and forgery. Therefore, finger vein recognition has recently attracted more and more attention in the research field of personal biometric identification [2-6].

However, finger vein NIR images are not always clear and there are a proportion of low-quality finger vein images in the captured images. The key issue in finger vein recognition is how to extract and represent features from NIR images effectively. Feature extraction becomes a critical step in the finger vein recognition procedure. Different methods, such as repeated line tracking [2], curvature method [3], minutiae descriptors [4], Gabor filters [5] and binary pattern based methods [6], etc., have been proposed for finger vein feature extraction. These existing methods can be roughly divided into two categories: global feature extraction and local feature extraction. The global feature extraction methods [2-3] are applied the geometric shape, topological structure, or curvature information from the segmented blood vessel network as finger vein features. However, the low quality of finger vein images may cause segmentation errors during the feature extraction procedure and degrade the performance of finger vein recognition [7]. The local feature extraction methods, including Local Binary Pattern (LBP) [4] and their variants [6-8], are applied to extract finger vein feature without blood vessel

*Corresponding Author 
segmentation. Although the local feature extraction methods can achieve better recognition performance than the global feature extraction methods, these works fail to consider the fact that finger vein is essentially a kind of network containing rich directional information.

To solve the above problems, a new approach is proposed in this paper for finger-vein recognition. Inspired by the work of [6], we find that Log Gabor filters have more powerful characteristics than Gabor filters in space frequency, space position and direction selectivity of finger vein global pattern. Moreover, LBP only represents the first order circular derivative pattern and fails to extract the high order discriminative information from finger vein local pattern. Conversely, Local Derivative Pattern (LDP) [9] can effectively reflect and quantize the local directional information hidden in finger vein local pattern. In this paper, the combination of Log Gabor filter and LDP, namely GLDP, is proposed to extract both the global structure and the local direction information from finger vein images. In addition, Blocked Principal Component Analysis (BPCA) method is introduced to reduce the dimensionality of the extracted GLDP feature vector and enhance its discriminability. The experimental results show that the proposed approach can significantly improve the recognition performance compared to other existing approaches.

The rest of the paper is organized as follows: Section 2 presents the proposed GLDP feature extraction approach. Section 3 describes the proposed finger vein recognition algorithms in detail. Section 4 presents the experimental results to verify the validity of the proposed approach. Finally, Section 5 concludes this paper.

\section{GLDP Feature Extraction for Finger Vein Image}

The framework of the proposed finger vein feature extraction approach based on GLDP is illustrated in Figure 1. In this approach, a finger vein image can be modeled as a feature vector by the following procedure:

(1) A normalized finger vein image is filtered by the multi-scale and multi-orientation Log Gabor filters to obtain the Log Gabor Magnitude Pattern (LGMP); (2) Each LGMP is divided into non-overlapping rectangle blocks with specific size, and further converted to Log Gabor Local Derivative Pattern (GLDP) by LDP operator; (3) The gray-level histogram of GLDP is computed in each block region; (4) The region histograms within all scales and all orientations are concatenated to form the final feature vector of finger vein image. The following subsections describe this procedure in detail.

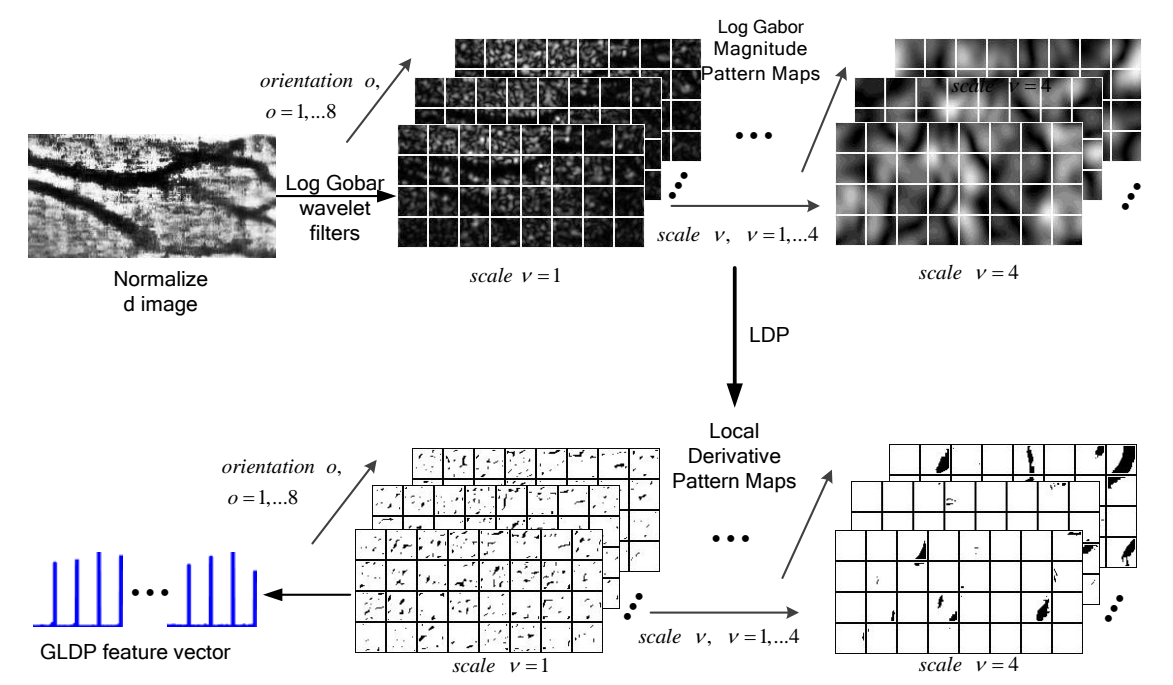

Figure 1. The Framework of the Finger Vein GLDP Feature Extraction 


\subsection{Log Gabor Magnitude Pattern}

Log Gabor filters [10] are proposed to overcome the bandwidth limitation in traditional Gabor filters. Compared to Gabor filters, Log Gabor filters have two special characteristics [10]. Firstly, there is no DC component in the Log Gabor transfer function. Secondly, the Log Gabor transfer function can be extended by a large range in frequency. In a polar coordinate system, 2D Log Gabor transfer function [11] can be defined as Equation (1):

$G(\rho, \theta)=e^{-\frac{\log ^{2}\left(\rho / u_{0}\right)}{2 \sigma^{2}}} e^{-\frac{\left(\theta-9_{0}\right)}{2 \sigma_{\theta}^{2}}}$

where $(\rho, \theta)$ represents the polar coordinates, $u_{0}$ is the filter's central frequency, $\theta_{0}$ is the orientation angle, $\sigma$ and $\sigma_{\theta}$ are the radial and angular bandwidth, respectively. Filter bandwidth is controlled by $\sigma$ and filter orientation is controlled by $\theta$. The real and imaginary parts of 2D Log Gabor filter shapes transformed into spatial domain are shown in Figure 2.

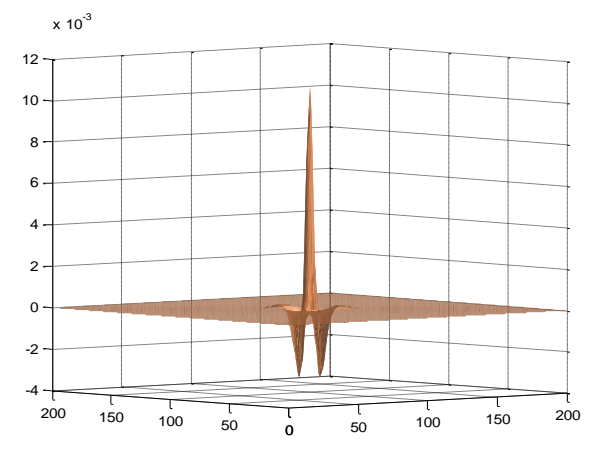

(a) Real part

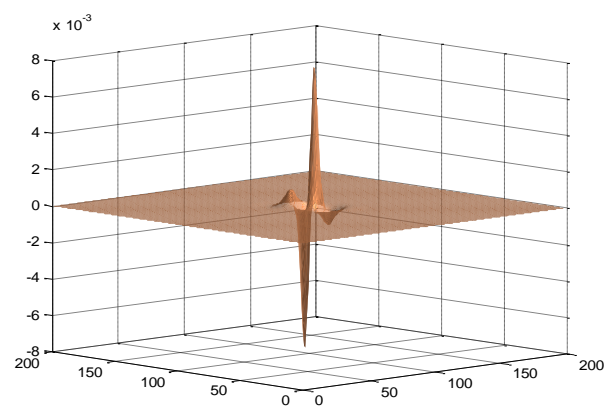

(b) Imaginary part

\section{Figure 2. The Illustration of 2D Log Gabor Filter Shapes in Spatial Domain}

In this paper, Log Gabor filters are applied with the following parameters: filter orientation $o \in\{0, \pi / 8,2 \pi / 8, \cdots, 7 \pi / 8\}$ and filter scale $s \in\{0, \ldots, 3\}$ (their corresponding centre frequencies are $1 / 3,1 / 6,1 / 12$, and $1 / 24$, respectively). The choice of four scales and eight directions can be guaranteed to obtain finger veins of different width and various directions in images. Let $I(Z)$ denote a normalized finger vein image, the convolution between image $I(Z)$ and Log Gabor filter $G_{s, o}(z)$ is defined as Equation (2):

$$
I_{s, o}(Z)=I(z) * G_{s, o}(z)
$$

where $G_{s, o}(z)$ is a Log Gabor filter with scale $s$ and orientation $o, I_{s, o}(Z)$ is the convolution result and symbol $*$ denotes the convolution operator. $I_{s, o}(Z)$ is composed of the real part $\operatorname{Re}_{s, o}(Z)$ and imaginary part $\operatorname{Img}_{s, o}(Z)$. Based on the two parts, the magnitude value $\operatorname{Mag}_{s, o}(Z)$ at each pixel position can be computed by Equation (3).

$$
\operatorname{Mag}_{s, o}(Z)=\sqrt{\operatorname{Re}_{s, o}^{2}(Z)+\operatorname{Img}_{s, o}^{2}(Z)}
$$

Each image is convolved with 32 Log Gabor filters in this paper, and 32 Log Gabor Magnitude Patterns (LGMP) are totally generated. 


\subsection{Local Derivative Pattern}

Local Derivative Pattern (LDP) operator [9] is a high order image texture descriptor. Compared to the first order directional local pattern extracted by LBP, LDP can extract the higher order derivative direction information in the local region. LDP encodes the variations of the neighborhood derivative direction, which can offer more discriminative information than LBP. Therefore, the second order LDP is introduced to calculate the derivative variation in LGMP.

The first order LDP operator of each pixel in an image is applied by subtracting its neighbor pixels according to the derivative directions along $0,45,90$ and 135 degrees. Let $I_{0}$ be a pixel in an image $I$, and $I_{j}, j=1, \cdots 8$ be the neighbor pixels around $I_{0}$ (see Figure 3).

\begin{tabular}{|l|l|l|}
\hline$/ 1$ & $l_{2}$ & $l_{3}$ \\
\hline$/ 8$ & 10 & $l_{4}$ \\
\hline$l_{7}$ & 16 & 15 \\
\hline
\end{tabular}

Figure 3. Eight Neighbor Pixels Considered in LDP

The four first order derivatives at $I_{0}$ can be calculated by Equation (4).

$$
\begin{aligned}
& I_{0^{\circ}}^{\prime}\left(I_{0}\right)=I\left(I_{0}\right)-I\left(I_{4}\right) \\
& I_{45^{\circ}}^{\prime}\left(I_{0}\right)=I\left(I_{0}\right)-I\left(I_{3}\right) \\
& I_{90^{\circ}}^{\prime}\left(I_{0}\right)=I\left(I_{0}\right)-I\left(I_{2}\right) \\
& I_{135^{\circ}}^{\prime}\left(I_{0}\right)=I\left(I_{0}\right)-I\left(I_{1}\right)
\end{aligned}
$$

The second order LDP operator in direction $\alpha=0^{\circ}, 45^{\circ}, 90^{\circ}, 135^{\circ}$ at $I_{0}$ can be defined as Equation (5).

$$
L D P_{\alpha}\left(I_{0}\right)=\left\{f\left(I_{\alpha}^{\prime}\left(I_{0}\right), I_{\alpha}^{\prime}\left(I_{1}\right)\right), f\left(I_{\alpha}^{\prime}\left(I_{0}\right), I_{\alpha}^{\prime}\left(I_{2}\right)\right), \cdots, f\left(I_{\alpha}^{\prime}\left(I_{0}\right), I_{\alpha}^{\prime}\left(I_{8}\right)\right)\right\}
$$

where $f()$ is a binary function defined as Equation (6).

$$
f\left(I_{\alpha}^{\prime}{ }_{\alpha}\left(I_{0}\right), I_{\alpha}^{\prime}\left(I_{i}\right)\right)=\left\{\begin{array}{l}
0, \text { if } I_{\alpha}^{\prime}\left(I_{0}\right) \cdot I_{\alpha}^{\prime}\left(I_{i}\right)>0 \\
1, \text { if } I_{\alpha}^{\prime}{ }_{\alpha}\left(I_{0}\right) \cdot I_{\alpha}^{\prime}{ }_{\alpha}\left(I_{i}\right) \leq 0
\end{array}, i=1, \cdots, 8\right.
$$

Thus, the second order LDP code of the given pixel $I_{0}$ in direction $\alpha=0^{\circ}, 45^{\circ}, 90^{\circ}, 135^{\circ}$ can be further obtained by Equation (7).

$$
\operatorname{LDPcode}_{\alpha}\left(I_{0}\right)=\sum_{i=1}^{8} f\left(I_{\alpha}^{\prime}\left(I_{0}\right), I_{\alpha}^{\prime}\left(I_{i}\right)\right) 2^{8-i}
$$

The second order LDP encoding result at a pixel $I_{0}$ in Log Gabor Magnitude Pattern of the image $I$ with scale $s$ and orientation $o$ is denoted as $\operatorname{LDPcode}_{\alpha}^{s, o}\left(I_{0}\right), \alpha=0^{\circ}, 45^{\circ}, 90^{\circ}, 135^{\circ}$. Finally, the proposed Log Gabor Local Derivative Pattern (GLDP) of the image $I$ is defined as a set of the four directional $\operatorname{LDPcode} e_{\alpha}^{s, o}\left(I_{0}\right)$ shown in Equation (8). 
$G L D P_{s, o}(I)=\left\{L D P \operatorname{code} e_{\alpha}^{s, o}\left(I_{0}\right) \mid \forall I_{0} \in I, \alpha=0^{\circ}, 45^{\circ}, 90^{\circ}, 135^{\circ}\right\}$

where $s$ and $o$ represent the corresponding scale and orientation of Log Gabor filter.

It can be seen from the above equations that the LDP operator encodes each pixel in LGMP of the image $I$ and generates $G L D P_{s, o}(I)$ codes consequently.

\subsection{GLDP Feature Representation}

Since the quality of finger-vein images is highly influenced by the imaging conditions [7], the feature representation of the proposed $G L D P_{s, o}(I)$ is summarized by the proposed Algorithm 1.

Algorithm.1 GLDP Feature Representation
Input: $I$, a input normalized finger vein image; $m$, the number of blocks for LGMP;
Log Gabor filters of orientation $o=0, \pi / 8,2 \pi / 8, \cdots, 7 \pi / 8$ and scale $s=0, \ldots, 3 ;$

Output: the feature vector $F$.

Step 1: For the image $I, I$ is convolved with Log Gabor filters of orientation $o$ and scale $s$ by Equation (1)-(3) to obtain 32 LGMPs;

Step 2: For each LGMP, the corresponding $L D P \operatorname{code} e_{\alpha}^{s, o}(I)$ is computed by Equation (4)-Equation (7), and each LGMP is spatially divided into $m$ non-overlapping blocks with specific size;

Step 3: For each LGMP, $L D P \operatorname{code} e_{\alpha}^{s, o}(I)$ in each block of the LGMP is binned to a gray histogram $h_{s, o, b}, b=1,2, \ldots, m$;

Step 4: For each LGMP, all $h_{s, o, b}$ are concatenated into a single histogram sequence $H_{s, o}=\left(h_{s, o, 1}, \ldots, h_{s, o, m}\right)$ to represent the given LGMP;

Step 5: Concatenating all $H_{s, o}, o=0, \pi / 8,2 \pi / 8, \cdots, 7 \pi / 8, s=0, \ldots, 3$, as the final feature vector $F$ to represent the image $I$;

Step 6: Output $F$.

Firstly, the image $I$ is convolved with Log Gabor filters of orientation $o \in\{0, \pi / 8,2 \pi / 8, \cdots, 7 \pi / 8\}$ and scale $s \in\{0, \ldots, 3\}$ to obtain 32 LGMPs. Secondly, the corresponding LDPcode $e_{\alpha}^{s, o}(I)$ in each LGMP is computed by Equation (4)-(7), and each LGMP is spatially divided into multiple non-overlapping blocks with specific size. Thirdly, $L D P \operatorname{code} e_{\alpha}^{s, o}(I)$ in each block of the LGMP is binned to a gray histogram $h_{s, o, b}$, $b \in\{1, \ldots, m\}$. Fourth, all $h_{s, o, b}$ are concatenated into a single histogram sequence $H_{s, o}$ to represent the given LGMP in the scale $s$ and orientation $o$. Finally, all $H_{s, o}$ are further concatenated as the final feature vector $F$ to represent the image $I$, where $F=\left(h_{0,0,1}, \ldots, h_{0,0, m}, h_{0,1,1}, \ldots, h_{0,1, m}, \ldots, h_{7,3,1}, \ldots, h_{7,3, m}\right)$.

\section{Finger Vein Recognition Based on GLDP Feature}

In this paper, the two approaches are applied to measuring the similarity between two GLDP feature sequences: the direct matching measurement and the Blocked PCA method.

\subsection{Direct Matching}

There are different measures used to provide the similarity metrics for histogram matching. The histogram intersection (see Equation (9)), performs slightly better than other direct matching metrics in the finger vein recognition. 


$$
D(p, q)=\frac{\sum_{i} \min \left(p_{i}, q_{i}\right)}{\sum_{i} q_{i}}
$$

In Equation (9), $p$ and $q$ respectively represent the two histograms, which are compared of each $i$ bins in histogram intersection. Using this measurement, the similarity of two GLDP feature sequences $F^{1}, F^{2}$ can be obtained by Equation (10).

$S\left(F^{1}, F^{2}\right)=\sum_{s=0}^{3} \sum_{o=0}^{7} \sum_{b=1}^{m} D\left(h_{s, o, b}^{1}, h_{s, o, b}^{2}\right)$

where

$$
F^{1}=\left(h_{0,0,1}^{1}, \ldots, h_{0,7, m}^{1}, h_{1,0,1}^{1}, \ldots, h_{1,7, m}^{1}, \ldots, h_{3,0,1}^{1}, \ldots, h_{3,7, m}^{1}\right)
$$

and

$F^{2}=\left(h_{0,0,1}^{2}, \ldots, h_{0,7, m}^{2}, h_{1,0,1}^{2}, \ldots, h_{1,7, m}^{2}, \ldots, h_{3,0,1}^{2}, \ldots, h_{3,7, m}^{2}\right)$.

Although the computation of Equation (10) is very efficient, the direct matching procedure is not good enough for the high feature dimension of GLDP representation. For example, when the number of $h_{s, o, b}$ histogram bins is $2^{8}=256$ and $m=32$, the dimension of $H_{s, o}$ is computed as $4 * 8 * 2^{8}=8192$ and the final dimension of feature vector $F$ is $32 * 8192=262114$. Due to the "small sample size" problem, Principal Component Analysis (PCA) algorithm [12] cannot be directly applied to reduce such high dimension of feature vector. Thus, the PCA approach based on blocks is alternatively presented.

\subsection{PCA Based on Blocks}

The basic idea of PCA based on blocks is to divide the high-dimensional GLDP feature vector into multiple segments (corresponding to different spatial blocks in LGMP), and then PCA algorithm is applied to each segment. The projected results of all the block-wise PCA (BPCA) are finally concatenated as the reduced feature vector. Since the dimension of the segmental sequences for PCA algorithm is much lower, the "small sample size" problem can be greatly weakened.

In PCA algorithm, all eigenvalues and eigenvectors are calculated and sorted during the training procedure of projection matrices. The eigenvectors corresponding to the top largest eigenvalues are chosen to construct the projective matrix, which projects the testing higher dimensional vectors into the lower dimensional space. Given the training vector set $\left\{x_{1}, x_{2}, \cdots, x_{m}\right\}$ and $\sum_{1}^{m} x_{i}=0$, the objective function of PCA is defined in Equation (11).

$J_{P C A}(W)=\underset{W}{\arg \max } W^{T} S_{t} W$

where $S_{t}$ is the covariance matrix of $x_{i}, i=1,2, \ldots, m$ and $S_{t}=\frac{1}{m} \sum_{i=1}^{m} x_{i} x_{i}^{T}$. Through solving the eigenvalues and eigenvectors of the covariance matrix $S_{t}$, PCA method seeks for the optimal projective matrix $W=\left[v_{1}, v_{2}, \cdots, v_{k}\right]$ according to the objective function defined in Equation (11), where $v_{1}, v_{2}, \cdots, v_{k}$ are the eigenvectors corresponding to the first $k$ largest eigenvalues of $S_{t}$. In the experiments, the value of $k$ is set to 0.9 .

Figure 4, illustrates the main procedure of GLDP feature dimensional reduction based on BPCA. For clarity, the procedure of BPCA projection and feature reduction are described in Algorithms 2 and 3, respectively. Thus, the workflow of the finger vein recognition based on BPCA algorithm is illustrated in Figure 5. This recognition approach includes two phases: enrollment and verification. In enrollment phase, 
finger vein images are normalized firstly, and then Log Gabor filters with LDP operator are applied to generate the GLDP feature vectors. All GLDP feature vectors of the enrollment samples are trained to obtain the projective matrices by Algorithm 2. The reduced feature vectors from the enrollment images are served as the reference templates by Algorithm 3. Both the projective matrices and the reference templates are stored in the database. During the verification phase, the projective matrices are taken out from the template database to reduce the dimension of the testing feature vectors by Algorithm 3. Finally, the matching score between the reduced dimension vectors and the reference templates is matched by Euclidean Distance.

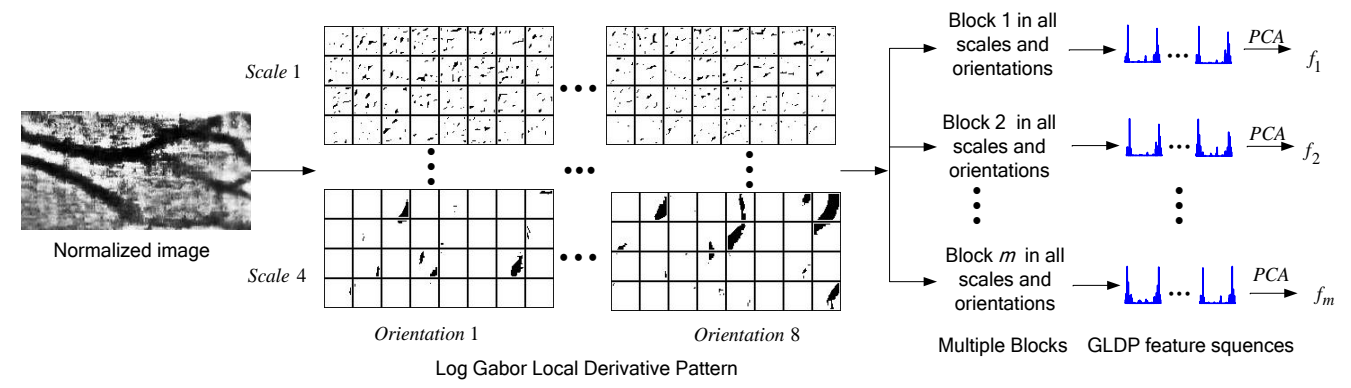

Figure 4. GLDP Feature Dimensional Reduction Based on BPCA

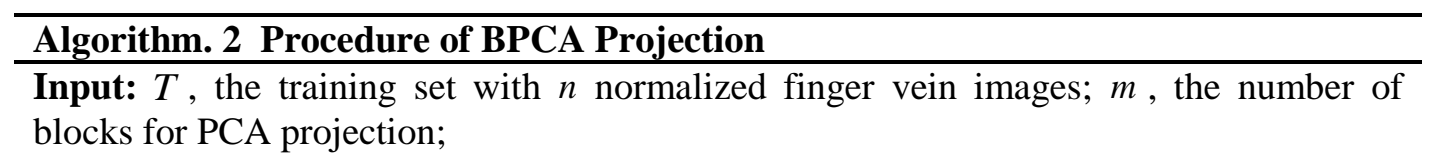

Output: the projective matrices $W_{i},(i=1,2, \ldots, m)$.

Step 1: For each image $I \in T$, according to Algorithm 1, computing its GLDP feature vector, $F=\left(h_{0,0,1}, \ldots, h_{0,7, m}, h_{1,0,1}, \ldots, h_{1,7, m}, \ldots, h_{3,0,1}, \ldots, h_{3,7, m}\right)$;

Step 2: For each block $i, i \in\{1, \cdots, m\}$ in $F$, concatenating its histograms of all scales and all orientations as one histogram sequence $H_{i}=\left(h_{0,0, i}, \ldots, h_{0,7, i}, h_{1,0, i}, \ldots, h_{1,7, i}, \ldots, h_{3,0, i}, \ldots, h_{3,7, i}\right)$.Thus, each image is represented as $m$ histogram sequences $H_{i},(i=1,2, \ldots, m)$;

Step 3: Obtain $m$ feature sets $S_{i}=\left\{H_{j, i} \mid j=1,2, \ldots, n, i=1,2, \ldots, m\right\}$ by collecting the features of the same spatial block from each of the $n$ training images, where $H_{j, i}$ denotes the histogram sequence from the $i^{\text {th }}$ block of the $j^{\text {th }}$ training image;

Step 4: For each block $i, i \in\{1, \cdots, m\}$, all $H_{j, i},(j=1,2, \ldots, n)$ is served as the training set to obtain the projection matrices $W_{i},(i=1,2, \ldots, m)$ according to Equation (11);

Step 5: Output the projective matrices $W_{i},(i=1,2, \ldots, m)$ into the template database.

\footnotetext{
Algorithm. 3 Feature Vector Reduction using Projection Matrices

Input: The normalized testing finger vein image $I, m$ and $W_{i}, \quad(i=1,2, \ldots, m)$ with the same meanings defined as Algorithm 2;

Output: The reduced feature vector $f$.

Step 1: For image $I$, its block-based representation $H_{i},(i=1,2, \ldots, m)$ is calculated by Steps 1-2 in Algorithm 2;

Step 2: For image $I$, its $i^{\text {th }}$ block low-dimensional vector $f_{i}$ is calculated by the linear
} 
transform $f_{i}=\left(W_{i}\right)^{T} H_{i}$, and concatenate all $f_{i},(i=1,2, \ldots, m)$ as the final reduced feature vector $f^{T}=\left[f_{1}^{T}, f_{2}^{T}, \ldots, f_{m}^{T}\right]$;

Step 3: Output the feature vector $f$.

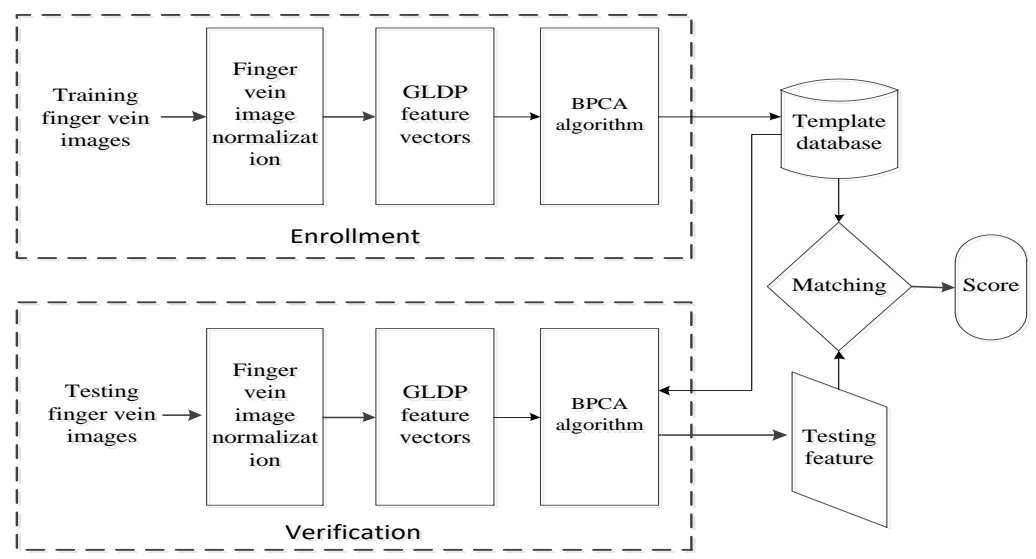

Figure 5. Finger Vein Recognition Workflow based on BPCA

\section{Experimental Results and Analysis}

\subsection{Experiment Setup}

The proposed finger vein recognition approach is evaluated on The Hong Kong Polytechnic University Finger Image Database [13]. The database consists of 3134 touchless NIR finger vein images with the resolution of $513 * 256$ pixels. There are randomly selected 100 fingers with six vein images per-subject for the recognition performance evaluation. In addition, the method [14] is applied to preprocess the finger vein images and normalize the region of interest $(\mathrm{ROI})$ with the fixed size $(128 * 256)$ pixels.

In the recognition experiments, 3 samples per-subject are used for the training procedure and 3 samples for the testing procedure. In order to decide how the parameters of the proposed algorithm affect the recognition performance, all the experiments are evaluated on the same test scale. The evaluation protocols of False Match Rate (FMR), False Non-Match Rate (FNMR), and Receiver Operator Characteristic (ROC) curve [15] are applied in this paper. The Equal Error Rate (EER), the point where FNMR is equal to FMR, is always used to evaluate the recognition performance in biometrics. Note that the lower value of EER shows the better recognition accuracy for biometric applications.

\subsection{Parameter Selection}

The transfer function of Log Gabor filters is designed with respect to the quadrature pair filters in the frequency domain [16]. In the implementation of Equation (1), the parameters are empirically tuned based on the criterion that the parameter values can lead to the lower EER. As a result, the parameters of Equation (1) are set as the following: there are eight filter orientation $\theta_{0} \in\{0, \pi / 8,2 \pi / 8, \cdots, 7 \pi / 8\}$ where the bandwidth of the filters are controlled by the parameter $\sigma_{\theta}=\{0.85,0.75,0.65,0.55\}$, and the central frequencies at four scales are set to $u_{0} \in\{1 / 3,1 / 6,1 / 12,1 / 24\}$. Due to the characteristics of the LDP operator, the size of block is set to be the power of 2 . In the experiments, the number of blocks is chosen by 16, 32, 64, and 128. In Algorithm 1, the number of histogram bins is set to 256 . 
The performance analysis of the proposed GLDP feature is presented in Figure 6. It illustrates the ROC curves' comparison between the direct matching method (GLDP Direct) and the BPCA method (GLDP+BPCA). As shown in Figure 6, EER results change with the number of blocks $(m=16,32,64,128)$. It can be found that EER of the BPCA method is superior to that of the direct matching method in the different number of blocks. Moreover, the proposed BPCA method has the stronger discrimination than the direct matching method. In this case, the BPCA method using 32 blocks $(\mathrm{GLDP}+\mathrm{BPCA} \mathrm{m}=32)$ results in the best EER performance. The reason is that the smaller block blocks cannot preserve the effective vein directional information while the distinction of vein pattern in the larger blocks is not remarkable. When the number of blocks $m$ is set to 32, it is a balance between the directional information preservation and the discriminating pattern to achieve the optimal recognition performance.

The performance of BPCA method with the change of scale $s$ is further analyzed when the orientation $o$ is fixed to $\{0, \pi / 8,2 \pi / 8, \cdots, 7 \pi / 8\}$ and the number of blocks $m$ is set to 32. From the Figure 7, it can be seen that the better EER performance can be obtain on scale 3 than on other single scales. However, the best EER can be obtained effectively when the Log Gabor filters with all scales are together exploited the various width of finger veins. The best EER performance of BPCA method can be calculated as $0.20 \%$ when the GLDP feature presentation is obtained by the four scales and eight directions of Log Gabor filters.

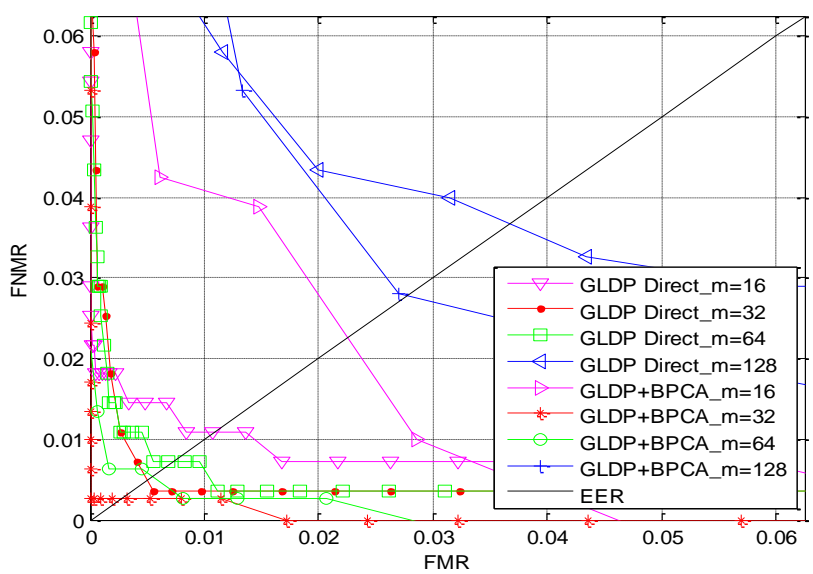

Figure 6. ROC Curves of Direct Matching Method and BPCA Method

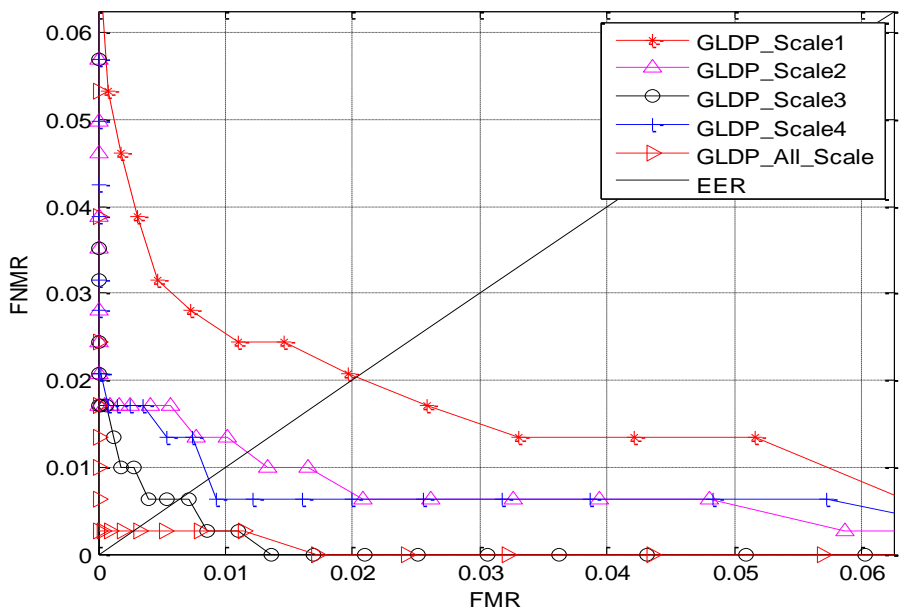

Figure 7. ROC Curves of GLDP Feature Using Different Scales 


\subsection{Comparable Results}

In order to show the superiority of GLDP feature representation in finger vein recognition, the proposed approach is also compared with other existing approaches. The comparable experiments are conducted on the same finger vein images. The comparable results in terms of EER are summarized in Table 1. From Table 1, it is observed that the recognition performance of the proposed method performs significantly better than the other approaches [2-5]. Besides, the recognition accuracy for GLDP is obviously better than GLBP [6].

In addition, Figure 8, shows the ROC curves of the different approaches in Table 1. For clear comparison, some lower performance methods are shown in Figure 8a, and the rest higher ones are shown in Figure 8b. From Figure 8, it can be seen that the proposed method has made the obvious improvement of recognition performance, which validates the effectiveness of the proposed GLDP feature representation.

Table 1. The EER Performance Comparisons between the Proposed Approach and Other Existing Approaches

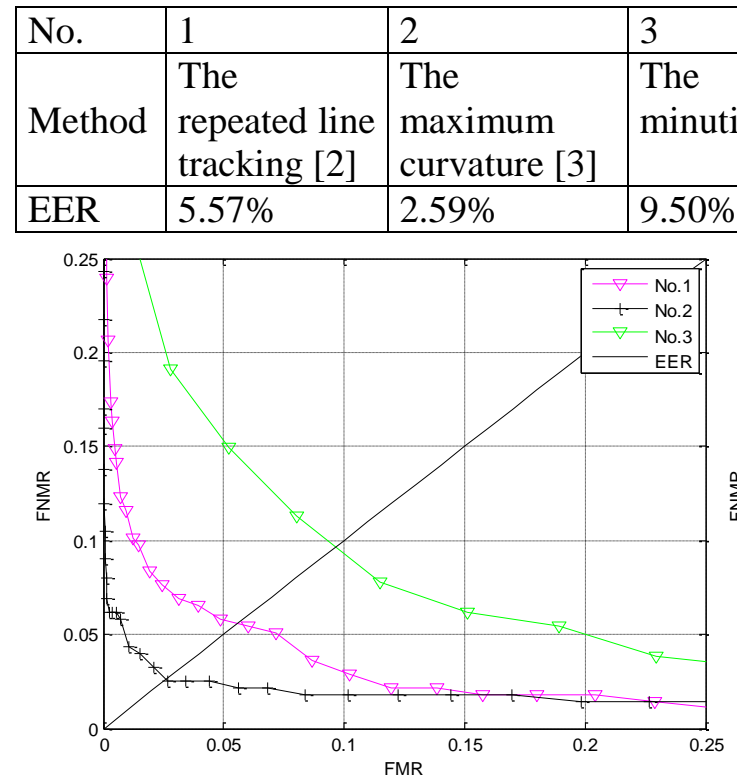

(a)

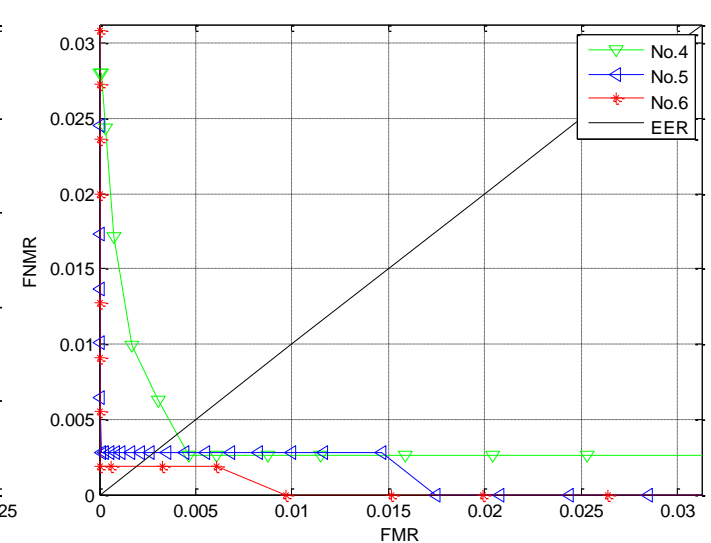

(b)

Figure 8. ROC Curves of Different Recognition Methods : (a) The Approaches with Lower Performance (b) The Approaches with Higher Performance

\section{Conclusion}

In this paper, a novel method for finger vein recognition method based on Log Gabor filter and Local Derivative Pattern are proposed. This method is composed of two main steps: GLDP feature extraction and BPCA. With LDP encoding technique, Log Gabor filters can generate more distinctive features in finger vein recognition. In addition, BPCA method is applied to reduce the dimensionality of the GLDP feature vector and improve recognition performance. Experimental results show that the proposed method has made significant improvements over other existing approaches. In future, we plan to fuse multiple features from the finger vein images to enhance the accuracy of finger vein recognition. 


\section{Acknowledgements}

This work is supported by Natural Science Foundation of Heilongiang Province of China (Grant No. QC2016091) and Scientific Research Fund of Heilongjiang Provincial Education Department (NO: 12531478).

\section{References}

[1] T. Yanagawa, S. Aoki and T. Ohyama, "Human finger vein images are diverse and its patterns are useful for personal identification", the 56th Session of the International Statistical Society, (2007) April 2.

[2] N. Miura, A. Nagasaka and T. Miyatake, "Feature extraction of finger-vein patterns based on repeated line tracking and its application to personal identification", Machine Vision and Applications, vol. 15, no. 4, (2004), pp. 194-203.

[3] N. Miura, A. Nagasaka and T. Miyatake, "Extraction of finger-vein patterns using maximum curvature points in image profiles", IEICE Transactions on Information \& Systems, vol. 90, no. D(8), (2007), pp. 1185-1194.

[4] E. C. Lee, H. C. Lee and K. R. Park, "Finger vein recognition using minutia-based alignment and local binary pattern-based feature extraction", International Journal of Imaging Systems and Technology, vol. 19, no. 3, (2009), pp. 179-186.

[5] A. Kumar and Y. Zhou, "Human identification using finger images", IEEE Transactions on Image Processing, vol. 21, no. 4, (2012), pp. 2228-2244.

[6] J. Peng, Q. Li, A. A. A. El-Latif, N. Wang and X. Niu, "Finger Vein Recognition with Gabor Wavelets and Local Binary Patterns", IEICE Transactions on Information and Systems, vol. E96, no. D(8), (2013), pp. 1886-1889.

[7] L. Yang, G. Yang, Y. Yin and R. Xiao, "Finger vein image quality evaluation using support vector machines", Optical Engineering, vol. 52, no. 2, (2013), pp. 027003-027009.

[8] L. Mirmohamadsadeghi and A. Drygajlo, "Palm vein recognition with local binary patterns and local derivative patterns", Proceedings of the 2011 International Joint Conference on Biometrics, (2011) October 11-13.

[9] B. Zhang, Y. Gao, S. Zhao and J. Liu, "Local derivative pattern versus local binary pattern: Face recognition with high-order local pattern descriptor", IEEE Transactions on Image Processing, vol. 19, no. 2, (2010), pp. 533-543.

[10] D. J. Field, "Relation between the statistics of natural images and the response properties of optical cells", The Optical Society of America A, vol. 4, no. 12, (1987), pp. 2379-2394.

[11] W. Wang, J. Li, F. Huang and H. Feng, "Design and implementation of log-gabor filter in fingerprint image enhancement”, Pattern Recognition. Letter, vol. 29, no. 3, (2008), pp. 301-308.

[12] M. Kirby and L. Sirovich, "Application of the Karhunen-Loeve procedure for the characterization of human faces", IEEE Transactions on Pattern Analysis and Machine Intelligence, vol. 12, no. 1, (1990), pp. 103-108

[13] "The hong kong polytechnic university finger image database version 1.0", http://www.comp.polyu.edu.hk/ csajaykr/fvtdatabase.htm, (2010).

[14] J. Peng, Q. Li, N. Wang, A. A. A. El-Latif and X. Niu, "An effective preprocessing method for finger vein recognition", The Fifth International Conference on Digital Image Processing, (2013) May 20-22.

[15] J. A. Unar, W. C. Seng and A. Abbasi, "A review of biometric technology along with trends and prospects", Pattern Recognition, vol. 47, no. 8, (2014), pp. 2673-2688.

[16] http://www.csse.uwa.edu.au/ pk/research/matlabfns/PhaseCongruency/Docs/convexpl.html, (2014).

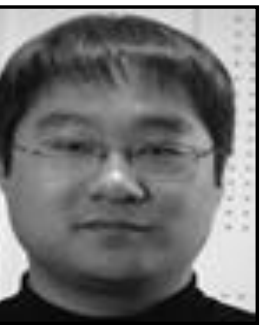

\section{Authors}

Lin Feng, was born in China, in September 1979. He received the M.S degree in Computer Technology in Harbin University of Science and Technology, Harbin, P. R. China in 2007. Now he is working in Information and Network Administration Center of Heilongjiang University. His research interests include biometric recognition, computer network, and web application. E-mail: linfeng@hlju.edu.cn. 


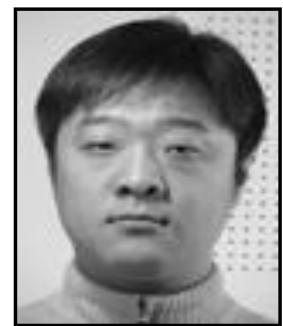

Wang Chao, was born in China, in March 1980. He received the M.S degree in Computer Technology in Harbin University of Science and Technology Harbin, P. R. China in 2007. Now he is working in Information and Network Administration Center of Heilongjiang University. His research interests include biometric recognition, computer network, and Network security. E-mail: wangchao@hlju.edu.cn.

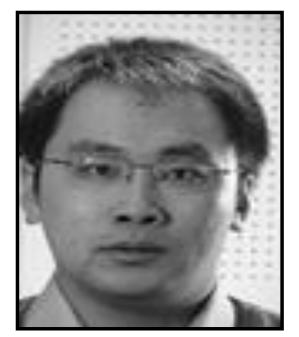

Peng Jialiang, was born in China, in January 1981. He received the Ph.d degree in Computer Science in Harbin Institute of Technology, Harbin, P. R. China in 2014. Now he is working in Information and Network Administration Center of Heilongjiang University. His research interests include biometric recognition, multi-biometric recognition, and information security. E-mail: pengjialiang@hlju.edu.cn. 